\title{
Rectal Invasion by Prostatic Adenocarcinoma That Was Initially Diagnosed in a Rectal Polyp on Colonoscopy
}

\author{
Ghilsuk Yoon · Man-Hoon Han ${ }^{1}$ \\ An $\mathrm{Na}$ Seo \\ Department of Pathology, School of Medicine, \\ Kyungpook National University, Kyungpook \\ National University Chilgok Hospital, Daegu; \\ ${ }^{1}$ Department of Pathology, School of Medicine, \\ Kyungpook National University, Kyungpook \\ National University Hospital, Daegu, Korea \\ Received: January 29, 2019 \\ Revised: March 5, 2019 \\ Accepted: March 25, 2019 \\ Corresponding Author \\ An Na Seo, MD, PhD \\ Department of Pathology, School of Medicine, \\ Kyungpook National University, \\ 90 Chilgokjungang-daero 136-gil, Buk-gu, \\ Daegu 41405, Korea \\ Tel: +82-53-200-3403 \\ Fax: +82-53-200-3399 \\ E-mail: san_0729@naver.com
}

\begin{abstract}
Despite anatomical proximity, prostatic adenocarcinoma with rectal invasion is extremely rare. We present a case of rectal invasion by prostatic adenocarcinoma that was initially diagnosed from a rectal polyp biopsied on colonoscopy in a 69-year-old Korean man. He presented with dull anal pain and voiding discomfort for several days. Computed tomography revealed either prostatic adenocarcinoma with rectal invasion or rectal adenocarcinoma with prostatic invasion. His tumor marker profile showed normal prostate specific antigen (PSA) level and significantly elevated carcinoembryonic antigen level. Colonoscopy was performed, and a specimen was obtained from a round, $1.5 \mathrm{~cm}$, sessile polyp that was $1.5 \mathrm{~cm}$ above the anal verge. Microscopically, glandular tumor structures infiltrated into the rectal mucosa and submucosa. Immunohistochemically, the tumor cells showed alpha-methylacyl-CoA-racemase positivity, PSA positivity, and caudal-related homeobox 2 negativity. The final diagnosis of the rectal polyp was consistent with prostatic adenocarcinoma. Here, we present a rare case that could have been misdiagnosed as rectal adenocarcinoma.
\end{abstract}

Key Words: Prostatic adenocarcinoma; Colonoscopy; Rectum; Polyp
Prostatic adenocarcinomas spread very slowly, although bone metastasis is common if the cancer is untreated. Prostatic adenocarcinoma with rectal invasion is extremely rare because of the rectoprostatic fascia (Denonvilliers' fascia) between the prostate and anterior rectal wall. ${ }^{1-5}$ Because of its rarity, prostatic adenocarcinoma with rectal invasion can be misdiagnosed as rectal adenocarcinoma on colonoscopic biopsy specimens. Here, we report a rare case of a patient with prostatic adenocarcinoma that was initially diagnosed from biopsy of a rectal polyp.

\section{CASE REPORT}

A 69-year-old Korean man presented with dull anal pain and voiding discomfort for several days in July 2018. His past medical history included diabetes mellitus treated with standard medications for several years. He showed no signs on the urinary system examination including digital rectal examination, prostate specific antigen (PSA) test and urinalysis a year ago, and he was relatively healthy until recently. Furthermore, he was relatively healthy, until recently. His recent tumor marker test results revealed the PSA level was within the normal limit at $3.25 \mathrm{ng} / \mathrm{mL}$ (normal range, 0.04 to $4.0 \mathrm{ng} / \mathrm{mL}$ ), and his carcinoembryonic antigen (CEA) level was significantly elevated at $18.26 \mathrm{ng} / \mathrm{mL}$ (normal range, 0 to $3 \mathrm{ng} / \mathrm{mL}$ ). A computed tomography scan revealed an abnormally enhancing mass in his entire prostate that extended into the anterior wall of the distal rectum and the urinary bladder, and multiple lymphadenopathy was also observed (Fig. 1A). Because the possibility of rectal adenocarcinoma with prostatic invasion could not be excluded, the patient underwent colonoscopy to distinguish between prostate cancer and rectal cancer. Colonoscopy revealed a protuberant, 1.5-cm-sized, sessile polyp that was located $1.5 \mathrm{~cm}$ above the anal verge (Fig. 1B). A microscopic examination of the rectal biopsy specimen revealed a glandular architecture with prominent nucleoli that infiltrated the rectal mucosa and submucosa (Fig. 2). However, no evidence of intraepithelial neoplasia was found in the rectal mucosa. Immunohistochemical staining demonstrated alpha-methylacyl-CoAracemase (P504s) positivity, PSA weak positivity, and caudal- 

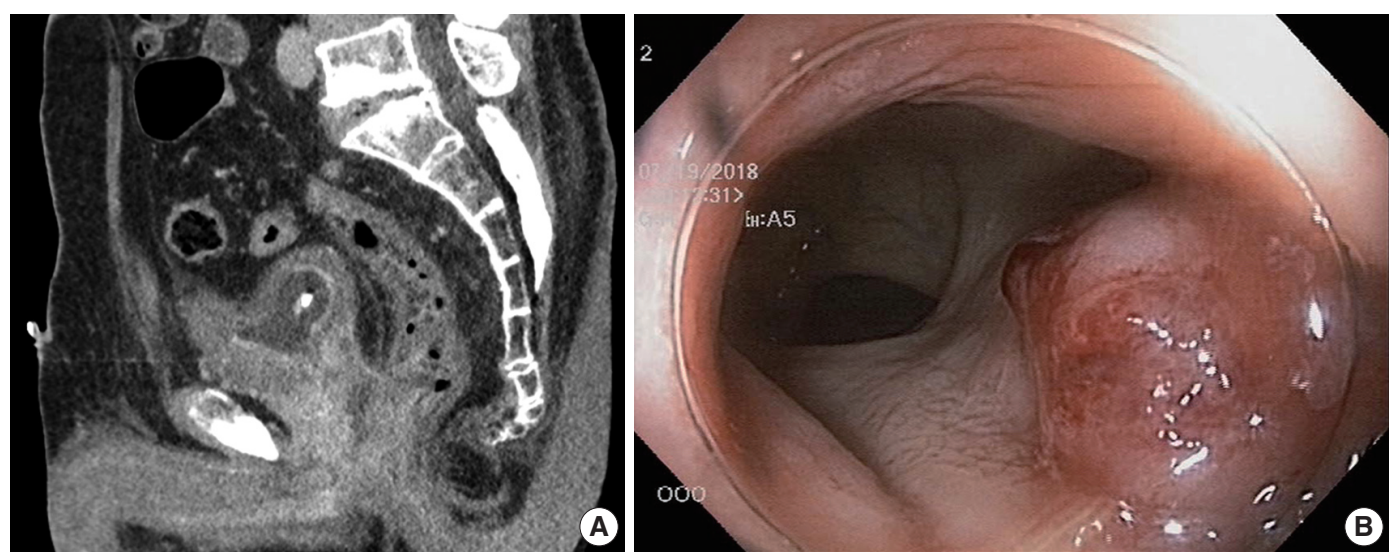

Fig. 1. (A) Abdominopelvic computed tomography scan reveals an abnormally enhancing mass in the prostate gland that invaded the urinary bladder, both seminal vesicles, and the anterior wall of the distal rectum. (B) Representative image of the colonoscopy shows a sessile rectal mass about $1.5 \mathrm{~cm}$ from the anal verge.
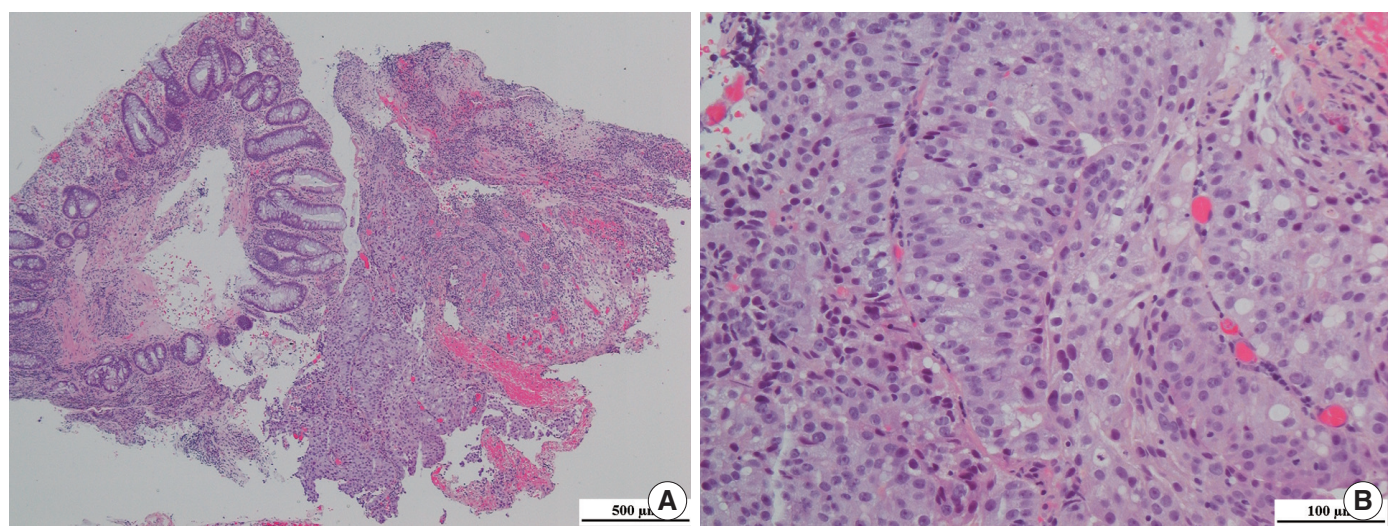

Fig. 2. (A) Representative image of hematoxylin and eosin staining of the rectal biopsy specimen. (B) Tumor cells had prominent nucleoli.

related homeobox 2 (CDX-2) negativity (Fig. 3). The tumor cells were consistent with a prostatic origin; thus, the final diagnosis of the rectal polyp was prostatic adenocarcinoma (Gleason score $4+4)$. After diagnosis of prostatic adenocarcinoma, the patient refused all procedures and treatment and was transferred to another hospital. Ethical approval was exempted for this case report by the Institutional Review Board of Kyungpook National University Medical Center, and the need for informed consent was waived through the de-identification of all the patient's personal information (No. KNUCH 2019-01-034).

\section{DISCUSSION}

Prostate cancer is a major contributor to cancer-related mortality and morbidity, despite its slow progression. ${ }^{6}$ Prostate cancer commonly metastasizes to the bones and lymph nodes, but metastases to the digestive tract are relatively rare. ${ }^{2}$ In addition, prostate cancer frequently directly infiltrates into the bladder and ureters but rarely invades the rectum due to Denonvilliers' fascia, a membranous barrier at the lowest part of the rectovesical pouch that separates the prostate and urinary bladder from the rectum and inhibits posterior spread of prostatic adenocarcinoma. Three potential routes have been proposed for invasion of prostate cancer into the rectal wall: (1) direct invasion through Denonvilliers' fascia; (2) lymphatic metastasis into the rectum; and (3) iatrogenic spread of cancer cells that seed into peri-rectal or rectal tissue along a needle biopsy tract. ${ }^{2,7}$ Our patient had no history of needle biopsy of the prostate. Unfortunately, prostate cancer with rectal invasion is occasionally confused with a primary rectal cancer with prostatic invasion because of their similar radiologic appearance, clinical presentation, and morphologic features. ${ }^{2,5}$ Notably, it might be difficult to differentiate prostatic adenocarcinoma from rectal adenocarcinoma in small rectal biopsy specimens. ${ }^{5,8}$ Tang et al. ${ }^{2}$ reported 9,504 patients, nine of whom had prostatic adenocarcinoma involving the rectal wall and were clinically and pathologically misdiagnosed with rectal adeno- 

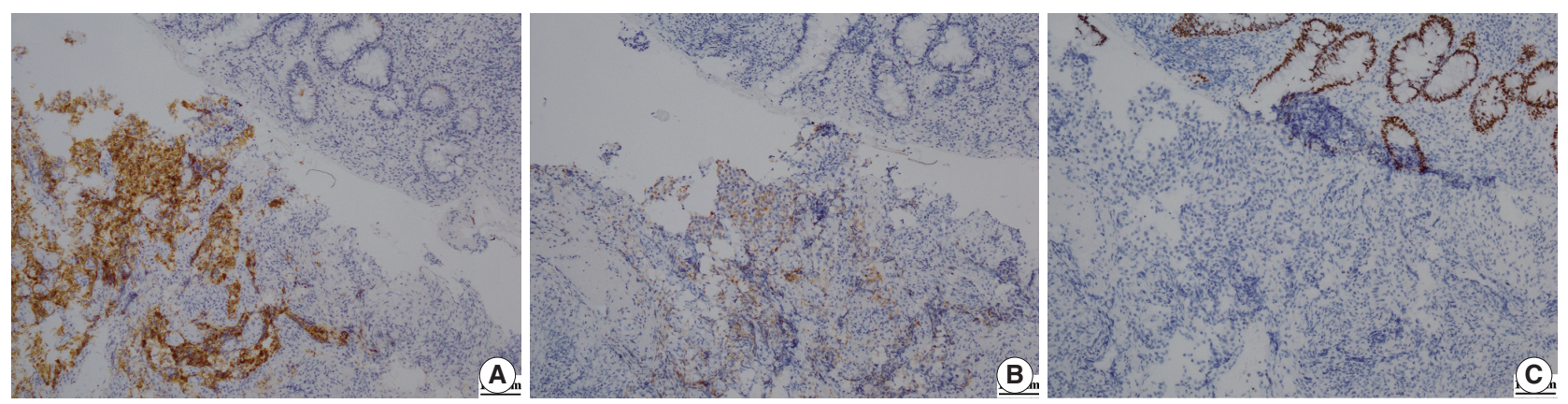

Fig. 3. (A) Immunohistochemical staining for alpha-methylacyl-CoA-racemase positivity shows strong cytoplasmic expression in tumor cells but not in the normal rectal crypt. (B) Immunohistochemical staining for prostate specific antigen shows weak expression in tumor cells but no expression in normal cells. (C) Immunohistochemical staining for caudal-related homeobox 2 shows strong nuclear expression in the normal rectal crypt but not in tumor cells.

carcinoma. This risk of misdiagnosis could result in inappropriate treatment strategies and lead to adverse consequences for the patient. Although PSA level might help distinguish these two entities, PSA is not expressed in all patients with prostatic adenocarcinoma. ${ }^{7,9}$ In our case, the PSA level was within normal limits, whereas the CEA level was significantly elevated, and the possibility of rectal adenocarcinoma could not be excluded.

Because several cases of extracolonic cancer have presented as colorectal polyps, consideration of a wide range of differential diagnoses is warranted. ${ }^{10}$ Delicate histologic differences between prostate and rectal cancers could facilitate accurate diagnoses. In the present case, the tumor consisted of small- or medium-sized glands with cribriform proliferation, and the cancer cells showed mild nuclear enlargement with prominent nucleoli. Unlike prostatic adenocarcinoma, the histologic features that favor rectal adenocarcinoma include tall columnar cells with basally located nuclei, 'dirty' necrosis, villous architecture, and stromal reaction. Furthermore, the immunohistochemistry of specific markers for these two entities helps confirm tumor origin. PSA and P504s are highly sensitive and specific commonly-used positive markers for prostatic adenocarcinoma. ${ }^{2,11,12}$ However, PSA and P504s are not always expressed in prostatic adenocarcinoma, and they show variable expression levels depending on tumor differentiation. ${ }^{13}$ Moreover, our patient showed weak PSA positivity but strong P504s positivity. In contrast, rectal adenocarcinoma commonly shows CDX-2 and CEA positivity but PSA and P504s negativity. Notably, some cases with androgen-independent prostatic adenocarcinoma displaying CDX-2 positivity have been reported,${ }^{14}$ demonstrating one of the potentially dangerous pitfalls for differentiating rectal adenocarcinoma. ${ }^{2}$ Conventionally, prostatic adenocarcinoma with rectal invasion represents an advanced stage and has a poor prognosis. ${ }^{5}$ In particular, rectal invasion is frequently a sign of more widespread systemic disease, which highlights the importance of a timely and accurate diagnosis. Above all, misdiagnosis as rectal adenocarcinoma can lead to inappropriate resection, such as abdominoperineal excision and anterior resection of the rectum.

In summary, we described a case of prostatic adenocarcinoma that presented with rectal symptoms and was initially diagnosed in a rectal polyp on colonoscopy. The patient had no previous medical history of prostatic adenocarcinoma, and his PSA level was within normal limits. Based on our experience, this rare finding might help clinicians and pathologists avoid misdiagnoses when faced with such cases in colorectal practice.

\section{ORCID}

Ghilsuk Yoon: https://orcid.org/0000-0002-9941-024X

Man-Hoon Han: https://orcid.org/0000-0001-8856-553X

An Na Seo: https://orcid.org/0000-0001-6412-3067

\section{Author Contributions}

Conceptualization: ANS.

Data curation: MHH.

Formal analysis: GY, ANS.

Funding acquisition: ANS.

Investigation: GY.

Methodology: GY, MHH, ANS.

Project administration: ANS.

Resources: ANS.

Writing—original draft: GY, ANS.

Writing—review \& editing: GY, MHH, ANS.

\section{Conflicts of Interest}

The authors declare that they have no potential conflicts of interest. 


\section{Acknowledgments}

We are indebted to Yong Hwan Kwon, MD, PhD of Kyungpook National University Chilgok Hospital (Daegu, South Korea) for the generous help with the colonoscopy.

\section{REFERENCES}

1. Lebret T, Méjean A. Rare locations of metastases from prostate cancer. Prog Urol 2008; 18 Suppl 7: S357-64.

2. Tang T, Yang Z, Zhang D, Qu J, Liu G, Zhang S. Clinicopathological study of 9 cases of prostate cancer involving the rectal wall. Diagn Pathol 2017; 12: 8.

3. Bowrey DJ, Otter MI, Billings PJ. Rectal infiltration by prostatic adenocarcinoma: report on six patients and review of the literature. Ann R Coll Surg Engl 2003; 85: 382-5.

4. Vaghefi H, Magi-Galluzzi C, Klein EA. Local recurrence of prostate cancer in rectal submucosa after transrectal needle biopsy and radical prostatectomy. Urology 2005; 66: 881.

5. Wang H, Yao Y, Li B. Factors associated with the survival of prostate cancer patients with rectal involvement. Diagn Pathol 2014; 9: 35.

6. Siegel RL, Miller KD, Jemal A. Cancer statistics, 2018. CA Cancer J Clin 2018; 68: 7-30.

7. Abbas TO, Al-Naimi AR, Yakoob RA, Al-Bozom IA, Alobaidly
AM. Prostate cancer metastases to the rectum: a case report. World J Surg Oncol 2011; 9: 56.

8. Lane Z, Epstein JI, Ayub S, Netto GJ. Prostatic adenocarcinoma in colorectal biopsy: clinical and pathologic features. Hum Pathol 2008; 39: 543-9.

9. Gallee MP, Visser-de Jong E, van der Korput JA, et al. Variation of prostate-specific antigen expression in different tumour growth patterns present in prostatectomy specimens. Urol Res 1990; 18: 181-7.

10. Steevens CD, Abraham J, Bahadur S. Metastatic prostate adenocarcinoma diagnosed in a colonic polyp. J Clin Oncol 2012; 30: e160-2.

11. Jiang Z, Woda BA, Wu CL, Yang XJ. Discovery and clinical application of a novel prostate cancer marker: alpha-methylacyl CoA racemase (P504S). Am J Clin Pathol 2004; 122: 275-89.

12. Oesterling JE. Prostate specific antigen: a critical assessment of the most useful tumor marker for adenocarcinoma of the prostate. J Urol 1991; 145: 907-23.

13. Cho KR, Epstein JI. Metastatic prostatic carcinoma to supradiaphragmatic lymph nodes: a clinicopathologic and immunohistochemical study. Am J Surg Pathol 1987; 11: 457-63.

14. Werling RW, Yaziji H, Bacchi CE, Gown AM. CDX2, a highly sensitive and specific marker of adenocarcinomas of intestinal origin: an immunohistochemical survey of 476 primary and metastatic carcinomas. Am J Surg Pathol 2003; 27: 303-10. 